\title{
THE EFFECT OF PRESSURE ON MAGNETIC INDUCTION.
}

By Fannie Cornelia Frisbie.

$\mathrm{T}$

$\mathrm{HE}$ reciprocal relations existing between the change in shape or size of a body and its magnetization are so striking that they excite immediate interest, and make a thorough investigation of these relations necessary before a satisfactory theoretical explanation of the phenomena of magnetization can be given. As different investigators of the effect of hydrostatic pressure on magnetic permeability have obtained contradictory results, it was thought worth while to undertake the problem in a new way. Before describing the method used it will be well to survey briefly the theoretical and the experimental work already done.

On the supposition that Poisson's theory of induced magnetism for rigid bodies in a vacuum is correct, Helmholtz ${ }^{1}$ ( $188 \mathrm{I}$ ) deduced, with the aid of the principle of conservation of energy, expressions for the forces which act between the particles of magnetic or dialectric polarized bodies. These agreed with those of Clerk Maxwell ${ }^{2}$ and Sir Wm. Thomson, except that they contained, beside the coefficient of induction, a constant which depends upon the nature of the medium, and which is determined by the change which it undergoes on change of density. Kirchoff ${ }^{3}$ (I 884) introduced another constant which makes it possible to express the change experienced by induction through dilations which are different in different directions, thus applying to solid bodies as well as to fluids.

Drude ${ }^{4}$ has shown from purely theoretical considerations that since the shape of a body is altered by magnetization, any alteration

1 Wied. Ann., r3, p. 400 ; Abhandlung, I., p. 798.

2 Electricity and Magnetism, § 644 .

${ }^{3}$ Wied. Ann., vol. 24, p. 52 ; Gesammelte Abhandlung, Nachtrag, p. 9 r.

${ }^{4}$ Physik des Aethers, p. ${ }^{56}$. 
of shape or size should change the value of the coefficient of magnetic permeability of the body.

It is found that change in the state of magnetism of a body alters its shape and size in various ways depending upon the strength of the magnetizing field. ${ }^{1}$ The effect is to increase the length of an iron rod or ring in small magnetizing fields, as the field is made stronger the increase reaches a maximum, falls to zero, and for still stronger fields there is a diminution in length. It is found that accompanying the increase in length there is a decrease in thickness, and this led the early investigators to conclude that there was no volume change due to magnetization; later experimenters ${ }^{2}$ have, however, found a rapid increase of volume with $H$, the magetizing force, to a certain point after which it increases asymptotically.

Since there was found to be a deformation of a body with an alteration of its magnetization, it was natural that one should next look for a reciprocal relation between the two phenomena, and it was found that a long thin rod under longitudinal tension underwent an increase of magnetization ${ }^{3}$ until the magnetic moment reached a certain amount, after which further pull produced a diminution. Longitudinal and also transverse pressure produced effects opposite to those of longitudinal stretch.

The effects of hydrostatic pressure were investigated (1882) by Wassmuth ${ }^{4}$ who placed the iron rod inside a thick walled tube which he filled with mercury. Pressure was obtained by the

1 Wiedemann, Lehre von der Electricität, III., pp. 703-714. Joule, Phil. Mag., (3) 30, pp. 76, 225, I847; reprint of papers, p. 235. Cantonne, Mem. d'R. Accad., vol. 6, p. 487 , I89o. Bidwell, Proc. Roy. Soc. , 40, pp. 109, 257, I886 ; 43, p. 406, 1888; 47, pp. 46-49, 189o ; 56, p. 94, I894; Phil. Trans., vol. I 79 A, I888, p. 205 ; Beiblätter, I 2, p. 600. Knott, Proc. Roy. Soc. Edin., 19, p. 249, I892 ; 20, pp. 290, 295, 334, I893-5 ; Trans. Roy. Soc. Edin., 38, p. 527, 1896.

2 Nagaoka and Honda, Phil. Mag., 5, vol. 37, p. 131, 1894; 5, vol. 46, p. 26r, I898; Beibl. I8, p. 595 ; Wied. Ann., vol. 53, p. 487, r894.

3 Wiedemann, Lehre von der Electricität, III., pp. 698-703. Wertheim, Compt. Rend., 35, p. 702, I852 ; Ann. de Chim. et Phys. (3), 50, p. 385, 1857. Matteucci, Ann. de Chim. et Phys. (3), 53, p. 416, 1858. Villari, Pogg. Ann., 126, p. 67, 1868. Sir Wm. Thomson, Phil. Trans., I66, p. 693, 1876; Beibl. 2, pp. 362, 607 ; Proc. Roy. Soc., 23, pp. 445, 473, $1875 ; 27$, p. 439, 1878 . Tomlinson, Proc. Roy. Soc. Lond., 42, p. 224, I887; Beibl. I I, p. 659. Ewing, Beibl., 13, p. 186; Phil. Trans., 179, 333, I888. Chree, Proc. Roy. Soc. Lond., 47, p. 4I, 1899; Beibl., I4, p. 398 ; I'll1. Trans., I $81 \mathrm{~A}$.

4 Wien. Ber., 86 , pp. 539, 55०, 1882; Beibl. 7, p. 43, 1883. 
expansion of the mercury when heated by means of warm water. One end of the glass tube was drawn out to a capillary from which increase of pressure was observed. The glass tube was surrounded by a solenoid which exerted a nearly uniform magnetizing force upon the iron rod. The intensity of magnetization was learned by the deflection of a mirror. He found that with increasing hydrostatic pressure the temporary moment in general decreased, and only increased for magnetizations which lie near the maximum. $\mathrm{He}$ estimated that he had about ten atmosphere pressure. Tomlinson ${ }^{1}$ (I887) investigated the same subject and obtained negative results. He found no perceptible change either in the magnetic permeability of soft iron or in the permanent magnetism of hard steel; but at the beginning or at the end of the hydrostatic pressure, as similarly with longitudinal compression and extension, a part of the residual magnetism vanished. Nagaoka and Honda again obtained positive results, but these were not in agreement with those of Wassmuth. They had two similar brass tubes containing the rods to be examined. About the tubes were wound the magnetizing solenoids. The tubes were filled with water and pressure was obtained by means of a Cailletet pump. A magnetometer placed symmetrically with regard to the rods was adjusted until there was no deflection, then pressure was applied to one, and the change in magnetization produced a deflection of the magnetometer which could be observed. They used rods and ovoids of soft iron and found a decrease in the magnetization with pressure up to 250 atmospheres, using a magnetizing field, $H$, from $5-5.5$ c.g.s. units. They calculated from data furnished by other parts of their series of experiments on magnetostriction the result to which Kirchoft's theory leads and found that the theory calls for an increase. Their series of experiments agreed with theory for the most part, but in regard to the effect of hydrostatic pressure there was not the same accord between experiment and theory. Unfortunately they make no statement about the condition of the iron and its previous treatment.

The present experiment may be divided into two parts : (I) The investigation of the effect of different pressures upon each of the rings with a constant magnetizing force; and also the influence: 
of residual magnetism upon the results. (2) The investigation of the effect of a certain pressure when magnetizing forces of different strengths are exerted upon the iron; and also of the variation of results with change in the condition of the iron.

It was desired to avoid all end effects by the use of anchor rings, to secure higher pressure than the other experimenters had used and to test the effects with the same ring under various conditions and in different rings of the same material.

To obtain the pressure a special form of apparatus was designed (see Fig. I) consisting of two parts of iron, $19 \mathrm{~cm}$. outside diameter,

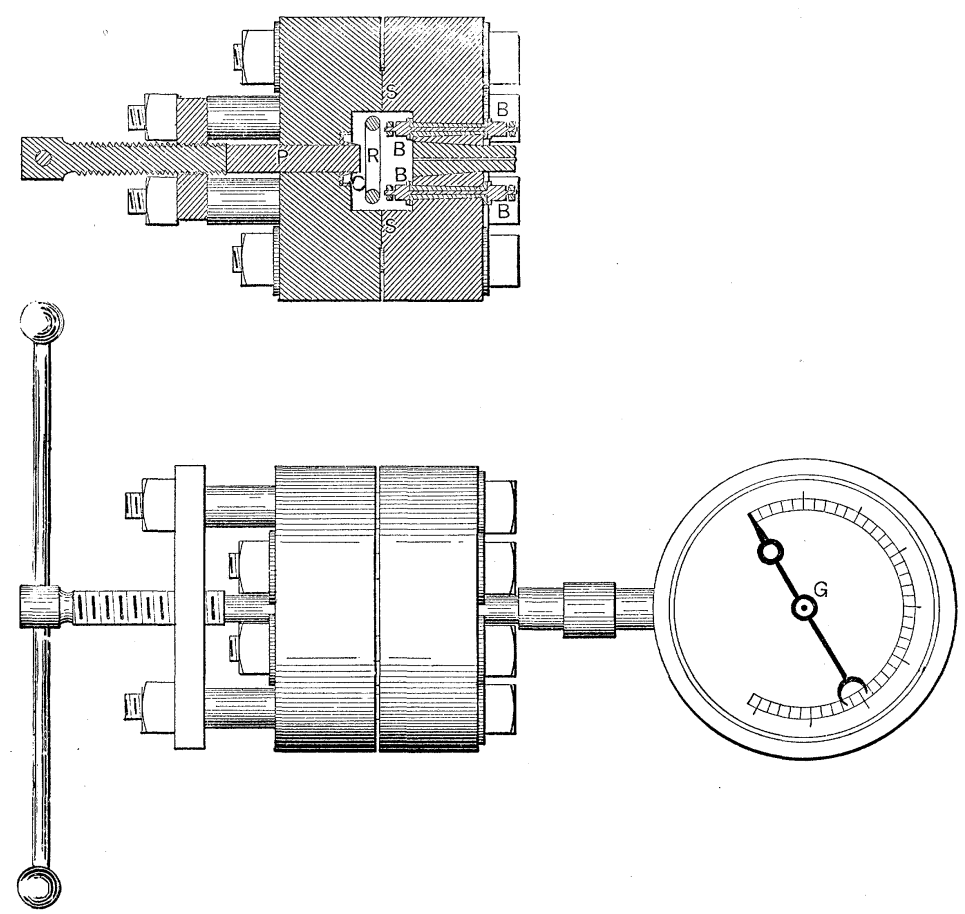

Fig. 1.

with walls $6.2 \mathrm{~cm}$. thick. The contact surfaces $(S)$ were well ground and the iron ring $(R)$ was placed within, then the two parts were joined by means of eight five-eighth-inch bolts, thus making a thickwalled compartment. The connection of wires was made by means of four metal plugs terminating on either side in binding posts $(B)$ 
and insulated by taper plugs of hard rubber which passed through one end of the cylinder. A plunger in the other end was the means of obtaining pressure and the cavity was filled with oil through the hole for the plunger. Resin oil was used, which besides being very viscous is an excellent insulator. To avoid air bubbles the cylinder was first exhausted by an aspirator pump and the oil was then forced in by atmospheric pressure. A copper cap $(C)$ prevented the leakage of oil around the plunger. Great difficulty was experienced in making the cylinders so tight that the desired pressure could be obtained without leakage. Various materials were triel between the halves but the tightest joint was finally secured by having the contact surfaces well ground. At first a slight smearing of vaseline and beeswax was used but later even this was found unnecessary. The pressure obtained was read directly from the gauge which registered as high as 18,000 lbs. per sq. in.

The three rings used were cut as nearly alike as possible from the same piece of Norway iron. They were wound with a secondary of No. 36 silk-covered copper wire, over which was a primary of No. 24 silk-covered copper wire. The layers were insulated from the iron and from one another by windings of insulating paper. Great care was exercised in winding that no wires should overlap. The dimensions and the data concerning the three rings used are given in the following table :

\begin{tabular}{l|c|c|c}
\hline & Ring I. & Ring II. & Ring III. \\
\hline Mean outer diameter. & $5.46 \mathrm{~cm}$. & $5.41 \mathrm{~cm}$. & $4.45 \mathrm{~cm}$. \\
Mean diameter of iron. & .624 & .623 & .661 \\
No. turns in primary. & 203 & 213 & 203 \\
No. turns in secondary. & 1,208 & 1,310 & 1,208 \\
\hline
\end{tabular}

The galvanometer used was a D'Arsonval ballistic.

Part 1. - Two rings were placed inside two pressure cylinders, so as to have both under the same conditions, and their primaries were connected in series with the desired number of cells of the storage battery, a resistance and an ammeter. The direction of throw of the galvanometer for each ring when the primary circuit was made or broken was noted; and the two secondaries were then connected in series with the galvanometer in such a way that the 
two throws opposed each other. It was then observed which coil was predominant in the effect and a series of readings was taken giving the throw for both make and break of the primary. The plunger of the cylinder was then pressed in by means of the screw until the desired amount was registered by the gauge and another series of readings was taken for the throw under the given pressure, after which a new set of zero pressure observations was recorded. The difference in the deflection of the galvanometer was an index of the change of induction due to the pressure exerted upon the iron core.

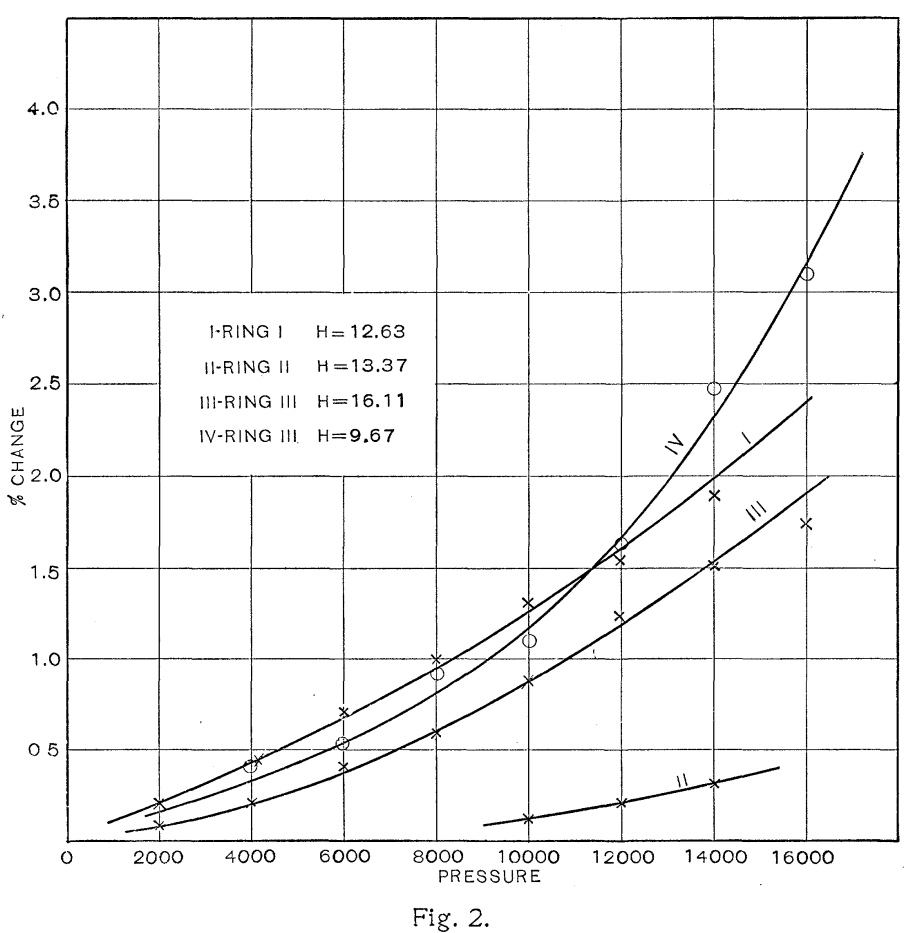

To ascertain the relative amount of this change the throw for each ring was observed when the same current was passed through the primary, and the secondary consisted of one or two turns; then the throw for the total number of turns, and the ratio of the change in induction to the total amount could be calculated.

In taking the readings it was found that the first throws after the 
pressure had been put on or removed were not in agreement with the later ones. This accords with Tomlinson's observation that part of the residual magnetism is lost when pressure is applied or removed. Before recording the deflections, the circuit was made and broken a sufficient number of times to secure a steady magnetic condition.

A correction must be made for the volume change due to the pressure. To make this the value for the modulus of elasticity of wrought iron $\left(=25 \times 10^{6}\right)$ given by Wm. Kent, Handbuch, p. 3 I 4 , was assumed and the correction made before the curves were plotted.

In Fig. 2, curves I., II., III., are shown the curves of the three rings, which represent the means of results of readings taken on different days. It is seen from the curves that although the percentage of change varies with the different pieces of iron, the effect of pressure is of the same general character for the three rings; and with a magnetizing force of from 12 to 16 absolute units, the magnetic permeability of iron in this condition is increased by hydrostatic pressure.

The following is a table of the results plotted in Fig. 2, curves I., II. and III. Centimeters change in deflection and the percentage this change is of the total deflection are given, also the corrected value, taking account of the variation of volume with pressure. The pressure is expressed in pounds per square inch. The magnetizing force, $H$, is in absolute units.

\begin{tabular}{|c|c|c|c|c|c|c|c|c|c|}
\hline \multirow[b]{2}{*}{ Pressure. } & \multicolumn{3}{|c|}{ I. $H=\mathrm{I} 2.63}$. & \multicolumn{3}{|c|}{ II. $H=\mathrm{I} 3.37}$. & \multicolumn{3}{|c|}{ III. $H=\mathrm{I} 6 . \mathrm{II}$} \\
\hline & $\mathrm{cm}$ & $\%$ & $\%$ cor. & $\mathrm{cm}$. & $\%$ & $\%$ cor. & $\mathrm{cm}$ & $\%$ & $\%$ cor. \\
\hline 2,000 & .18 & .20 & .21 & & & & .08 & .09 & .10 \\
\hline 4,000 & .36 & .40 & .42 & & & & .18 & .20 & .22 \\
\hline 6,000 & .62 & .68 & .70 & & & & .35 & .39 & .41 \\
\hline 8,000 & .86 & .95 & .98 & & & & .51 & .56 & .59 \\
\hline 10,000 & 1.15 & 1.27 & 1.31 & .08 & .08 & .12 & .76 & .84 & .88 \\
\hline 12,000 & 1.35 & 1.49 & 1.54 & .18 & .17 & .22 & 1.07 & 1.18 & 1.23 \\
\hline 14,000 & 1.68 & 1.84 & 1.90 & .26 & .25 & .31 & 1.32 & 1.46 & 1.52 \\
\hline 16,000 & & & & & & & 1.52 & 1.68 & 1.74 \\
\hline
\end{tabular}

To be sure that the change in the amount of the throw was not due to any alteration of temperature caused by the pressure, the 
plunger was screwed in and the whole apparatus allowed to stand until any temperature difference would have been equalized by conduction, and the readings then taken agreed with the others.

The use of anchor rings made it possible to avoid end effects, but the method was open to the objection that the pressure was exerted upon the windings as well as upon the iron core itself, and it was necessary to be assured that the effect was not caused by this. A brass ring of the same dimensions as those of iron described above, and with similar windings on it, was subjected to the same treatment as the iron rings, but no change of deflection was observed. To test it in another way a balance of the throws was obtained by the insertion of an auxiliary solenoid, and then pressures of ten thousand and of fourteen thousand pounds were applied to the brass ring, and still no deflection of the galvanometer was observed. This showed that the change in deflection found before was not due to any changes produced in the windings or in the insulation by pressure.

Experiments proved that the position of the plunger within the ring had no effect upon the amount of throw, and that the number of layers of insulating paper between the successive layers of wire was immaterial, and also that the change in the compressed ring was independent of the opposing one and the second coil served only to cut down the deflection caused by the other so that the change could be observed.

It was known throughout the previous observations that the iron was probably almost saturated with magnetism, and for that reason one ring was carefully demagnetized by a gradually diminishing alternating current, and a new series of observations was made with a smaller value of $H$ which would not give so near the maximum value of the intensity of magnetization. Great care was taken to keep the current steady as a slight change in the magnetizing force would cause a decided change in the magnetization. A change was also made in the method as the throw of the coil was diminished by resistance inserted in the secondary circuit instead of by means of a second coil. Each set of observations was taken with the current through the galvanometer first direct and then reversed. Curve IV., Fig. 2, shows the results obtained for ring III. after it had 
been demagnetized and treated in this manner, which shows that when the iron is not saturated with magnetism the percentage change is greater.

Results plotted in curve IV., Fig. 2, are given in the following table, where pressure is in pounds per square inch and the percentage change observed as well as the value corrected for volume change is recorded. Ring III. was used after demagnetization and $H=9.67$.

\begin{tabular}{c|c|c}
\hline Pressure. & Per cent. Change. & Per cent. Corrected. \\
\hline 4,000 & .39 & .41 \\
6,000 & .51 & .53 \\
8,000 & .89 & .92 \\
10,000 & 1.06 & 1.10 \\
12,000 & 1.58 & 1.63 \\
14,000 & 2.42 & 2.48 \\
16,000 & 3.05 & 3.11 \\
\hline
\end{tabular}

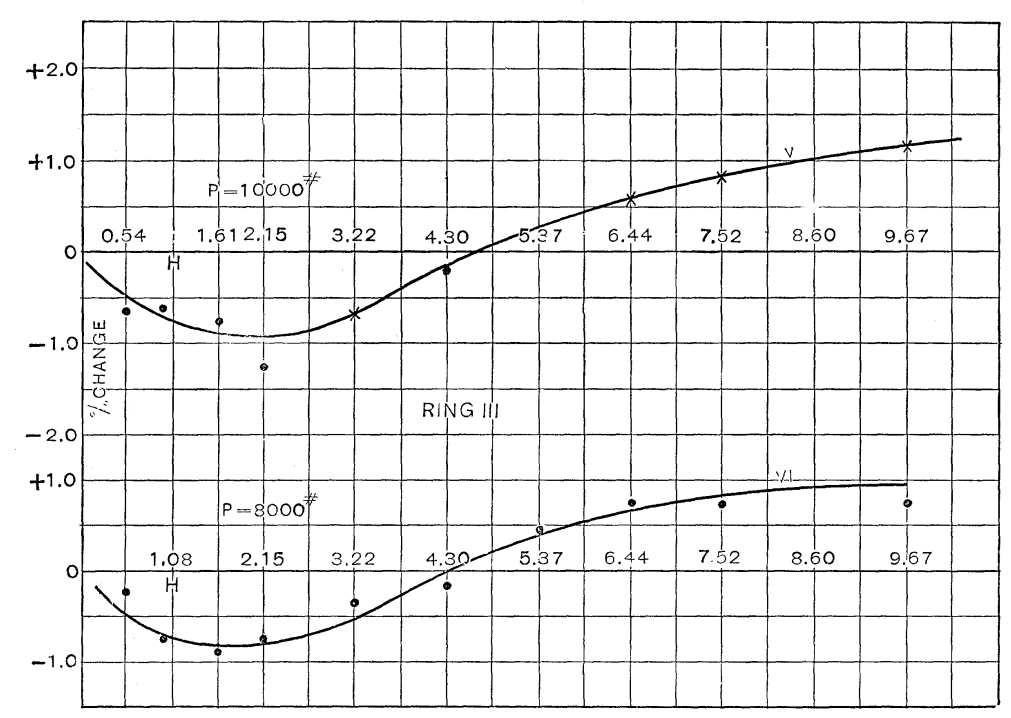

Fig. 3.

Part II. - As in the last observations of Part I. only one ring was used, and the deflection was cut down by the insertion of resistance in the secondary circuit. Instead of using the same current and taking the changes in deflection for various pressures, the current was varied for each set of readings and the pressure was 
each time made the same. The results are shown in Fig. 3, curves V. and VI. V. shows the percentage change of magnetic induction produced in ring III. by pressure of ten thousand pounds Table for Fig. 3. Curves V. and VI.; Current in Amperes and Values of $H$ in Absolute Units.

\begin{tabular}{|c|c|c|c|c|c|}
\hline \multirow{2}{*}{ Amperes. } & \multirow{2}{*}{$H$} & \multicolumn{2}{|c|}{ Curve V. ro, ,00 Pounds. } & \multicolumn{2}{|c|}{ Curve VI. 8,000 Pounds. } \\
\hline & & $\%$ Change. & $\%$ Corrected. & $\%$ Change. & $\%$ Corrected. \\
\hline .025 & .54 & -.67 & -.63 & -.24 & -.21 \\
\hline .045 & .97 & -.66 & -.62 & -.76 & -.73 \\
\hline .075 & 1.61 & -.77 & -.73 & -.90 & -.87 \\
\hline .100 & 2.15 & -1.31 & -1.27 & -.78 & -.75 \\
\hline .150 & 3.22 & -.71 & -.67 & -.36 & -.33 \\
\hline .200 & 4.30 & -.19 & -.15 & -.18 & -.15 \\
\hline .250 & 5.37 & +.05 & +.09 & +.41 & +.44 \\
\hline .300 & 6.44 & +.56 & +.60 & +.73 & +.76 \\
\hline .350 & 7.52 & +.78 & +.82 & +.69 & +.72 \\
\hline .450 & 9.67 & +1.11 & +1.15 & +.70 & +.70 \\
\hline
\end{tabular}

per square inch, when the magnetizing force varied from .54 to 9.67 c.g.s. units. VI. represents the effect of eight thousand pounds pressure for the same ring after demagnetization.

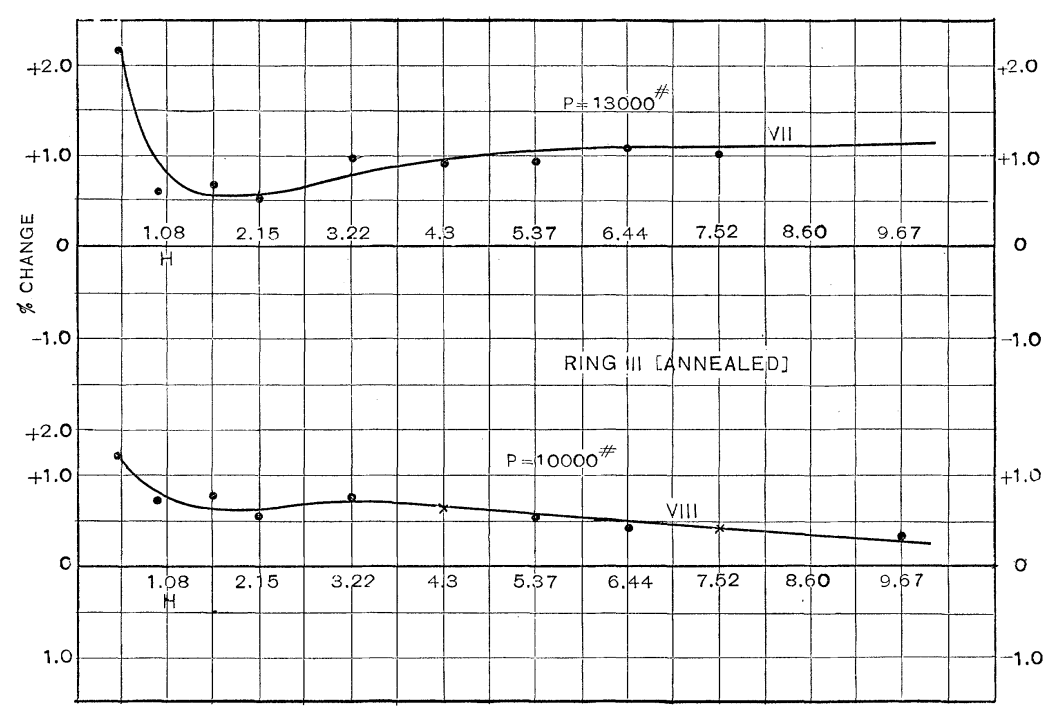

Fig. 4.

It is seen in both cases that with a small magnetizing force the magnetic induction decreases, reaching a minimum for a field 
whose strength lies between I.6 and 2.I absolute units. This diminution grows less with increasing fields, until for a value of $H$ about 4.5 or 4.8 it shows a zero change. Further strengthening of the magnetizing field results in an increase, which seems to approach a maximum for $H$ equal to approximately 9 c.g.s. units with eight thousand pounds pressure, and for a slightly larger value when the pressure is ten thousand pounds.

After the ring had been very carefully annealed another series of observations gave the results represented in curves VII. and VIII., Fig. 4. For VII., as the iron had just been annealed, there was no need of demagnetization; the pressure applied was thirteen thousand pounds per square inch. Before the readings for VIII. were taken the ring was demagnetized; the pressure in this case was ten thousand pounds. The table of results plotted is the following:

\begin{tabular}{c|c|c|c|c|c|c}
\hline \multirow{2}{*}{ Amperes. } & $H$ & \multicolumn{2}{|c|}{ Curve VII. } & 13,000 Pounds. & Curve VIII. & ro,ooo Pounds. \\
\cline { 3 - 5 } & & \% Change. & \% Corrected. & \% Change. & \% Corrected. \\
\hline .025 & .54 & +2.10 & +2.15 & +1.14 & +1.18 \\
.045 & .97 & .54 & .59 & .68 & .72 \\
.075 & 1.61 & .64 & .69 & .74 & .78 \\
.100 & 2.15 & .46 & .51 & .55 & .59 \\
.150 & 3.22 & .94 & .99 & .70 & .74 \\
.200 & 4.30 & .89 & .94 & .63 & .67 \\
.250 & 5.37 & .90 & .95 & .51 & .55 \\
.300 & 6.44 & 1.05 & 1.10 & .37 & .41 \\
.350 & 7.52 & .97 & 1.02 & .37 & .41 \\
.450 & 9.67 & & & .32 & .36 \\
\hline
\end{tabular}

It is interesting to note on comparison of these values with those obtained for the same ring before it was annealed, that in this case the change is an increase for every value of $H$ used, and that, with the exception of the first value which was obtained with a very small current, the percentages of change are included within narrower limits for the annealed iron. It is seen that the second, third and fourth points of the curve seem to give a slight wave, but it was felt that the results were hardly sufficiently accurate to warrant drawing it in. Curiously the effect of ten thousand pounds pressure diminishes for greater strengths of the field.

Throughout the observations great care was taken to keep the conditions steady. The current was watched carefully, and was 
maintained at the desired value by a slight alteration of the resistance in the primary circuit. The pressure was kept as constant as possible during the time required for the observations.

The experiment seems to warrant the following conclusions :

I. The application of hydrostatic pressure to soft iron anchor rings changes the coefficient of magnetic permeability of the iron, besides altering the amount of residual magnetism when the pressure is applied or removed. The amount of the change varies for different pieces of iron.

2. With unannealed iron the sign of the change depends upon the strength of the magnetizing field; the effect in small fields being a decrease, and in stronger fields an increase; the change of sign occurs for $H=4.5$ or 5 c.g.s. units. The magnitude of the alteration is a function of both field and pressure.

3. With annealed iron an increase is found for pressure with all values of field between .54 and 9.67 absolute units.

The results with the unnealed iron are in agreement with those obtained by Wassmuth in his experiment, as there is a decrease and then an increase for values of $H$ approaching the maximum of magnetization. But for the values of the field used by Nagaoka and Honda there is clearly an increase for iron, annealed and unannealed whereas they obtained only a decrease in magnetization, which was not the result called for by Kirchoff's theory and their own experiments on other forms of magnetostriction. The results of this investigation would then tend to support the theory of magnetism advanced by Kirchoff for annealed soft iron.

The author wishes to express her indebtedness to Professor Michelson, of the University of Chicago, for suggesting the problem, and the method of attack, and for his aid throughout the investigation; to Professors Mann and Millikan for their encouragement and assistance during the work ; and to Dr. Jewett who assisted in taking the final observations, and made the drawings for this article.

Barnard College. 